\title{
Equidad de género en las orquestas profesionales de Colombia
}

\section{Gender Equality in Professional Colombian Orchestras}

\author{
Laura Galindo Morales ${ }^{1}$
}

\section{Resumen}

Las preocupaciones por la equidad de género han sido constantes en los escenarios sociales de Colombia; la música, por supuesto, no constituye la excepción. El estudio de la participación de mujeres en las orquestas profesionales, los testimonios de las dos únicas directoras en la historia de la música sinfónica colombiana y las percepciones de un grupo de instrumentistas profesionales permiten entrever que, si bien se ha recorrido un camino significativo en las luchas por la equidad, la hegemonía patriarcal y el machismo bajo el cual estuvo regida la sociedad colombiana durante más de un siglo hacen que la meta aún esté lejos.

\section{Palabras claves}

Equidad de género, música, orquestas profesionales, significados intrínsecos, significados evocados, perfil musical masculino, discriminación, imagen.

\section{Abstract}

Concerns about gender equality have been a mainstay in the social landscapes of Colombia and music, of course, is no exception. The study of women's participation in professional orchestras, the testimonies of the only two female directors in the history of Colombian symphony music, and the perceptions of a group of professional instrumentalists, validates the idea that even though they have come a long way in the fight for equality, thanks to the patriarchal hegemony and machismo under which Colombian society has been ruled for more than a century, there is still a long way to go to achieve this goal.

\section{Key words}

Gender equality, music, professional orchestras, intrinsic meanings, evoked meanings, the masculine musical profile, discrimination, image.

Artículo recibido el 20 de octubre de 2014 y aprobado el 7 de abril de 2015

1 Universidad EAFIT, Medellín, Colombia. Correo electrónico: Igalind1@eafit.edu.co 


\section{Introducción}

Para el Programa de las Naciones Unidas para el Desarrollo (PNUD), la igualdad entre los géneros es un aspecto de justicia social y un asunto fundamental de derechos humanos, que supone la construcción de relaciones equitativas entre hombres y mujeres y la valoración de su aporte a la sociedad (Programa de las Naciones Unidas para el Desarrollo, s. f.). Si bien este es un concepto presente en el mundo occidental, respaldado por políticas legales en la mayoría de los países, ${ }^{2}$ sigue siendo una de las preocupaciones más grandes en la actualidad.

Según la escritora y jurista Alda Facio, especialista en asuntos de género y derechos humanos de las mujeres, las diferencias entre los sexos no implican desigualdad de ninguna índole y es posible concebir a hombres y mujeres como legalmente iguales en su mutua diferencia; sin embargo, a través de la historia no ha sido así. La desigualdad podría haberse dado en contra del sexo masculino si el parámetro de lo humano hubiera sido el inverso, pero pruebas empíricas demuestran que la jerarquización se hizo y se hace a favor de los varones, pues como Facio (2000) sostiene: "En la mayoría abrumadora de las culturas conocidas, las mujeres somos consideradas de alguna manera o en algún grado, inferiores a los hombres".

El surgimiento de esa desigualdad y jerarquización se reconoce en los estudios feministas y de género como "modelo patriarcal", que, en palabras de Ann Oakley (Sex, gender and society 1972), supone la inferioridad biológica de las mujeres con respecto a los hombres, asumiendo desde la antigüedad clásica y a través de disciplinas como la filosofía, la medicina, el derecho y la antropología, que las mujeres son física y mentalmente inferiores. Tal pensamiento se ha perpetuado con el pasar del tiempo y, hoy en día, la subordinación femenina se hace evidente en diferentes ámbitos: afectivos, sexuales, económicos y políticos; y en diferentes instituciones: la familia, el estado, la religión, las artes y la ciencia.

290 de los 177 países pertenecientes a al PNUD han informado de programas específicos de género.
En la música, las primeras ideologías patriarcales surgieron en el siglo Iv, cuando la Iglesia desaconsejó la práctica musical para las mujeres no pertenecientes a una orden religiosa, lo que se reafirmó en el Concilio de Trento, con la prohibición de cualquier tipo de educación formal para ellas; así, la polifonía, el uso de instrumentos distintos a la voz y las ejecuciones en público les fueron vedadas. Hacia finales del siglo XVIII, y durante el siglo XIX, instrumentos como el piano, el arpa y algunos otros de cuerda pulsada, como la guitarra y el laúd, se hicieron comunes entre las mujeres, siempre y cuando tuvieran fines privados y domésticos (Ramos, 2003).

En Colombia, el piano se hizo bastante popular entre las señoritas de clase alta, según lo afirma Juan Fernando Velásquez (2002). Leer, escribir, coser y tocar piano se volvieron parte indispensable de su educación, sin dejar, por ello, de ser inferior a la educación musical que podía recibir un hombre. Esta situación corresponde a lo que la musicología de género y Susan Green han llamado "el perfil masculino de la música” (2001). Para Green, la figura masculina ha sido asociada con "la mente" y la figura femenina con "el cuerpo", dando por supuesto una connotación negativa y de subordinación a la última. Actividades intelectuales como componer, interpretar y dirigir, fueron durante mucho tiempo tareas masculinas e inconcebibles en manos de una mujer.

Este fenómeno puede comprobarse a través de la evolución de los conjuntos y demás organizaciones musicales similares. Según estudios publicados por Pilar Ramos durante el 2003, desde el siglo vi $\mathrm{y}$ hasta la segunda mitad del siglo xx tocar en conjuntos estaba prohibido para las mujeres, más aún, si estos se presentaban en público y con fines lucrativos. Las únicas responsabilidades aceptadas socialmente para las mujeres de la época eran el manejo del hogar y la crianza de los hijos. Las monjas, libres de cualquier compromiso con el hogar debido a sus votos de castidad y celibato, eran las únicas que tenían permitida la práctica musical en público y en conjunto, siempre y cuando fuera de orden clerical y estuviera alejada de la práctica de los varones. 
Según Green (2001), para 1890 se crearon orquestas femeninas destinadas a tocar en cafés, cines o restaurantes, con fines recreativos, y disociadas del concepto de la orquesta profesional establecido en el clasicismo, donde la práctica sinfónica reconocida como oficial, primero en las cortes y más tarde en las ciudades, era exclusivamente masculina. Dichas orquestas de mujeres fueron relegadas en importancia, no reconocidas como profesionales y destinadas a la interpretación de repertorios ligeros.

Siguiendo con lo expuesto por Green, terminada la Segunda Guerra Mundial y como consecuencia del masivo reclutamiento de varones mayores de edad, las orquestas sinfónicas profesionales tuvieron un gran número de bajas en sus filas, lo que las llevó a buscar reemplazos para los instrumentistas faltantes en las orquestas conformadas por mujeres. $\mathrm{Al}$ ser consideradas como músicos de segunda categoría, la discriminación hacia las nuevas integrantes no se hizo esperar. Los directores, siempre en desacuerdo y obligados a aceptarlas por necesidad, se negaron a contratarlas de forma permanente y los demás músicos de la orquesta no dejaron de verlas como soluciones temporales que empobrecían el nivel de la orquesta.

Aunque la evolución de los tiempos ha ido desvirtuando esta idea, sus rezagos son evidentes. Un ejemplo célebre es el de la Orquesta Filarmónica de Viena que, considerada como una de las mejores y más grandes del mundo con cerca de 140 integrantes, solo ha aceptado a siete mujeres entre sus músicos desde el momento de su fundación (1842). Según un estudio informal realizado por Robert M. Levine en torno a las ICSOM Orchestras (International Conference of Symphony and Opera Musicians) durante 1993, cerca del $50 \%$ de los músicos profesionales en el mundo son mujeres; sin embargo, las orquestas europeas y estadounidenses están conformadas en un $84 \%$ y $70 \%$ por hombres, respectivamente. De 427 orquestas profesionales existentes en los Estados Unidos, solo 27 tienen a una mujer como directora titular $\mathrm{y}$, a pesar de presentar exactamente los mismos requisitos de audición, solo una cuarta parte de las orquestas tiene mujeres como jefes de fila en los primeros violines, mientras la mitad de ellas sí las tiene en los segundos (Levine, 2009).

Los anteriores no son eventos aislados, responden a un problema profundo que habita la práctica musical desde sus inicios y que hasta hace pocas décadas ha sido señalado. La crítica feminista ha sido más tardía en los estudios sobre música que en otras disciplinas, tuvo un primer antecedente en 1948 con la publicación de Music and Women por Sophie Drinker, pero no es sino hasta 1970 que este tipo estudios se vuelven regulares (Ramos, 2003). Al igual que en el feminismo anglosajón, Ramos establece tres etapas para el desarrollo de la musicología de género: la primera de ellas ha sido siempre la búsqueda de figuras femeninas en la música (intérpretes, compositoras, docentes y directoras); la segunda corresponde a la lucha en contra de la discriminación y la segregación de la que han sido víctimas; y la tercera es el estudio de los aspectos sicológicos que las caracterizan junto con las reacciones positivas o negativas que conllevan, entre ellas, la inequidad de género. Partiendo de los estudios realizados por autores dedicados a la musicología en Colombia, se podría considerar que el desarrollo feminista y de género en el entorno musical nacional se encuentra en algún punto entre la segunda y la tercera etapa.

A raíz de las anteriores consideraciones, y partiendo de que la Constitución política de Colombia garantiza la equidad de género en el artículo 43 del título 2: "La mujer y el hombre tienen iguales derechos y oportunidades. La mujer no podrá ser sometida a ninguna clase de discriminación", el presente estudio analiza la situación social de las mujeres en la música clásica colombiana, tomando como referencia las cinco orquestas profesionales reconocidas por el Ministerio de Cultura en la actualidad, a saber: Orquesta Filarmónica de Bogotá, Orquesta Sinfónica Nacional de Colombia, Orquesta Filarmónica de Medellín, Orquesta Sinfónica EAfIT y Orquesta Filarmónica de Cali.

\section{Metodología}

En este trabajo se asumió un método de investigación cualitativa aplicado desde dos diferentes pers- 
pectivas: la presencia de mujeres en las orquestas sinfónicas profesionales de Colombia y las experiencias de dichas mujeres en el ámbito laboral. Para el primer caso, se hizo un recuento del número de mujeres vinculadas a las cinco orquestas profesionales durante los últimos 10 años; y para el segundo, se recogieron las experiencias de las directoras Carmen Moral y Cecilia Espinosa, únicas titulares en la historia del país, y de ocho instrumentistas vinculadas formalmente a la Orquesta Filarmónica de Medellín y a la Orquesta Sinfónica EAfit.

\section{Descripción de las perspectivas}

- Participación de mujeres en orquestas:

Las cifras obtenidas surgen de una muestra base conformada por cinco orquestas que el Ministerio de Cultura, en conjunto con la Nueva Asociación Sinfónica Colombiana, han reconocido como únicas profesionales del país, a saber: la Orquesta Filarmónica de Bogotá, creada en 1967 y auspiciada por la Secretaría de Cultura del Distrito Capital; la Orquesta Sinfónica Nacional de Colombia, creada en el año 2002 luego de que el sistema de privatizaciones liquidara a su antecesora, la Orquesta Sinfónica de Colombia; la Orquesta Filarmónica de Medellín, fundada en 1983 por Alberto Correa Cadavid; la Orquesta Sinfónica EAFIT, que surge en el año 2002 gracias al patrocinio de la Universidad EAfIT; y la Orquesta Filarmónica de Cali, administrada por la Asociación para la Promoción de las Artes (Proartes) desde el año 2002.

- Experiencia de mujeres en orquestas:

a. Directoras: Desde el año 1952, momento en que se fundó la primera orquesta profesional en Colombia, solo dos mujeres han ejercido como directoras titulares en la música sinfónica colombiana: la peruana Carmen Moral, quien dirigió la Filarmónica de Bogotá entre 1988 y 1991, y la colombiana Cecilia Espinosa, quien se ha desempeñado como directora de la Sinfónica EAfit desde el momento de su creación. b. Instrumentistas: Se aplicó un cuestionario a ocho mujeres pertenecientes a la Orquesta Filarmónica de Medellín y la Orquesta Sinfónica EAFIT, con el propósito de establecer generalidades y particularidades en torno a temas de género, así como discriminación e influencia de la imagen en el ejercicio de la música profesional.

\section{Procedimiento}

- Participación de mujeres en orquestas:

Entre el 5 de noviembre y el 11 de diciembre del año 2013, fueron visitadas las sedes de las cinco orquestas mencionadas anteriormente, para determinar el porcentaje de mujeres que han participado de la música clásica profesional en Colombia. En dichas visitas se fotografiaron los programas de mano correspondientes a los conciertos sinfónicos realizados en los últimos diez años (2004-2013). Por cada año fueron elegidos los programas pertenecientes a los meses de marzo, junio y noviembre; de la información registrada como "planta artística", se tuvo en cuenta el número de mujeres con contrato laboral permanente. La elección de los meses responde al deseo de obtener un panorama general de la planta artística en cada año y poder compararlos entre sí.

- Experiencia de mujeres en orquestas:

a. Directoras: Con base en la propuesta de entrevista mixta descrita por María Galeano (Diseño de proyectos en la investigación cualitativa, 2004), el 13 de noviembre y el 6 de diciembre del año 2013, se realizaron entrevistas cualitativas a las directoras Carmen Moral, la cual tuvo lugar en Bogotá, aprovechando su visita para dirigir la Orquesta Filarmónica de esta ciudad; y Cecilia Espinosa, en el Departamento de Música de la Universidad EAFIT de Medellín.

Las dos entrevistas se hicieron a manera de charla informal y estuvieron delineadas por los siguientes temas: 
- Primera experiencia como directora titular: año, repertorio y orquesta.

- Experiencias personales en torno a la discriminación de género en la música.

- Opiniones y reflexiones sobre la participación de mujeres en la música sinfónica colombiana.

b. Instrumentistas: Siguiendo los parámetros establecidos por Jorge Padua (Técnicas de investigación aplicadas a las ciencias sociales, 1987), se elaboró un cuestionario de código múltiple en el que se incluyeron preguntas de tipo abierto y cerrado. Dicho cuestionario fue aplicado durante los meses de abril y mayo del año 2014, y tuvo como muestra a ocho instrumentistas de las orquestas sinfónicas profesionales de Medellín.

De cada orquesta fueron seleccionadas cuatro mujeres, tres que interpretan instrumentos de la familia de las cuerdas frotadas y una, de la familia de instrumentos de viento madera. El criterio para seleccionar a las encuestadas se deriva de los porcentajes obtenidos durante la etapa de la investigación previamente descrita. El cuestionario constó de nueve preguntas orientadas hacia las repercusiones de género en el momento de construir una carrera en la música clásica profesional.

\section{Resultados}

- Participación de mujeres en orquestas:

El número de mujeres en la planta artística de las orquestas colombianas es proporcional al número de integrantes de la misma, de la siguiente manera: entre mayor es el número de músicos, mayor es el número de mujeres en sus filas (tabla 1). Sin embargo, el porcentaje de mujeres con contrato laboral permanente se encuentra alrededor del $30 \%$ para las cinco orquestas de la muestra, y se ha mantenido dentro de este margen durante los últimos diez años (tabla 2).

Tabla 1. Número de mujeres instrumentistas durante los últimos diez años

\begin{tabular}{|c|c|c|c|c|c|c|c|c|c|c|}
\hline \multirow{2}{*}{$\begin{array}{l}\text { Año } \\
2004\end{array}$} & \multicolumn{2}{|c|}{$\begin{array}{l}\text { Filarmónica } \\
\text { de Medellín }\end{array}$} & \multicolumn{2}{|c|}{ Sinfónica de EAFIT } & \multicolumn{2}{|c|}{$\begin{array}{l}\text { Filarmónica } \\
\text { de Bogotá }\end{array}$} & \multicolumn{2}{|c|}{$\begin{array}{l}\text { Sinfónica } \\
\text { Nacional }\end{array}$} & \multicolumn{2}{|c|}{$\begin{array}{l}\text { Filarmónica } \\
\text { de Cali }\end{array}$} \\
\hline & Total & Mujer & Total & Mujer & Total & Mujer & Total & Mujer & Total & Mujer \\
\hline 2005 & 60 & 15 & 56 & 15 & 98 & 30 & 55 & 15 & 38 & 9 \\
\hline 2006 & 56 & 14 & 66 & 23 & 87 & 30 & 59 & 18 & 37 & 9 \\
\hline 2007 & 59 & 16 & 55 & 16 & 92 & 31 & 57 & 17 & 40 & 8 \\
\hline 2008 & 61 & 18 & 59 & 21 & 92 & 30 & 62 & 17 & 37 & 9 \\
\hline 2009 & 65 & 19 & 51 & 21 & 97 & 31 & 53 & 14 & 36 & 10 \\
\hline 2010 & 66 & 20 & 44 & 21 & 95 & 30 & 55 & 17 & 35 & 9 \\
\hline 2011 & 68 & 19 & 50 & 22 & 95 & 33 & 53 & 16 & 35 & 11 \\
\hline 2012 & 67 & 18 & 50 & 21 & 95 & 33 & 59 & 16 & 37 & 8 \\
\hline 2013 & 67 & 19 & 52 & 18 & 96 & 34 & 64 & 19 & 49 & 11 \\
\hline
\end{tabular}


Universidad Pedagógica Nacional

Facultad de Humanidades

Tabla 2. Porcentaje de mujeres instrumentistas durante los últimos diez años

\begin{tabular}{|c|c|c|c|c|c|}
\hline Año & $\begin{array}{c}\text { Filarmónica } \\
\text { de Medellín }\end{array}$ & Sinfónica EAFIT & $\begin{array}{c}\text { Filarmónica } \\
\text { de Bogotá }\end{array}$ & $\begin{array}{c}\text { Sinfónica } \\
\text { Nacional }\end{array}$ & $\begin{array}{c}\text { Filarmónica } \\
\text { de Cali }\end{array}$ \\
\hline 2004 & $25 \%$ & $27 \%$ & $30 \%$ & $20 \%$ & $23,5 \%$ \\
\hline 2005 & $25 \%$ & $35 \%$ & $34 \%$ & $30,5 \%$ & $24 \%$ \\
\hline 2006 & $27 \%$ & $29 \%$ & $34 \%$ & $30 \%$ & $20 \%$ \\
\hline 2007 & $30 \%$ & $41 \%$ & $33 \%$ & $28 \%$ & $24 \%$ \\
\hline 2008 & $30 \%$ & $41 \%$ & $32 \%$ & $27 \%$ & $28 \%$ \\
\hline 2009 & $30 \%$ & $48 \%$ & $32 \%$ & $31 \%$ & $31 \%$ \\
\hline 2010 & $28 \%$ & $44 \%$ & $36 \%$ & $30 \%$ & $21,5 \%$ \\
\hline 2011 & $27 \%$ & $42 \%$ & $36 \%$ & $27 \%$ & $21,5 \%$ \\
\hline 2012 & $29 \%$ & $35 \%$ & $35 \%$ & $30 \%$ & $22,5 \%$ \\
\hline 2013 & $27 \%$ & $27 \%$ & $34 \%$ & $28 \%$ & $19,5 \%$ \\
\hline
\end{tabular}

La orquesta que presenta mayores cambios en este porcentaje es la Orquesta Sinfónica EAfIT, que para el año 2009 estuvo a punto de igualar el número de hombres y mujeres en sus filas. Sin embargo, esta cifra ha ido disminuyendo durante los últimos años, acomodándose así a la regularidad presentada por la muestra. La Orquesta Filarmónica de Cali, por su parte, se encuentra en el otro extremo: el porcentaje de mujeres entre sus músicos de planta pocas veces superó el $25 \%$, la participación femenina más baja dentro de las cinco orquestas estudiadas.

Con excepción de la Orquesta Sinfónica EAfIT, cuya naturaleza será analizada más adelante, nin- guna de las otras cuatro orquestas presenta una variación mayor al $10 \%$ en su número de mujeres, lo que demuestra que la participación femenina en la música sinfónica colombiana es mucho menor a la masculina.

Del total de mujeres vinculadas a las cinco orquestas profesionales en Colombia entre los años 2004 y 2013 , cerca del $70 \%$ lo ha estado en la familia de los instrumentos de cuerda, entre el $7 \%$ y el $24 \%$ en la familia de las maderas, entre el $4 \%$ y el $9 \%$ en la percusión y menos del $5 \%$ en los instrumentos de viento metal. Una vez más, para este último caso la excepción es la Orquesta Sinfónica EAfit (tabla 3).

Tabla 3. Porcentaje de mujeres por familias de instrumentos

\begin{tabular}{|c|c|c|c|c|c|}
\hline $\begin{array}{l}\text { Familia de } \\
\text { Instrumentos }\end{array}$ & $\begin{array}{l}\text { Filarmónica } \\
\text { de Medellín }\end{array}$ & Sinfónica EAFIT & $\begin{array}{l}\text { Filarmónica } \\
\text { de Bogotá }\end{array}$ & $\begin{array}{l}\text { Sinfónica } \\
\text { Nacional }\end{array}$ & $\begin{array}{l}\text { Filarmónica } \\
\text { de Cali }\end{array}$ \\
\hline Cuerdas & $72 \%$ & $68 \%$ & $70 \%$ & $79 \%$ & $80 \%$ \\
\hline Maderas & $24 \%$ & $12 \%$ & $16 \%$ & $7 \%$ & $21 \%$ \\
\hline Metales & - & $13 \%$ & $3 \%$ & $5 \%$ & - \\
\hline Percusión & $4 \%$ & $7 \%$ & $8 \%$ & $9 \%$ & $9 \%$ \\
\hline Piano & - & - & $3 \%$ & - & - \\
\hline
\end{tabular}

Vale la pena aclarar que el piano no figura como instrumento de planta en la mayoría de las orquestas y que la participación de los instrumentistas corres- pondientes está subordinada a que el montaje en curso lo requiera. Solo la Orquesta Filarmónica de Bogotá y la Orquesta Sinfónica Nacional han vincu- 
lado pianistas de forma permanente, Olga Trouchina y Sergei Sichkov, respectivamente. Por tal razón, no es posible establecer un porcentaje de participación femenina para este caso.

\section{Consideraciones sobre la Orquesta Sinfónica EAFIT}

La Orquesta Sinfónica EAfit fue creada en el año 2000, a partir de una propuesta presentada por Hilda Olaya y Cecilia Espinosa, durante la rectoría de Juan Felipe Gaviria, quien acogió la idea de generar una nueva oferta musical para la ciudad.

La Orquesta está conformada, en su mayoría, por egresados, estudiantes y docentes del Departamento de Música, razón que explica los porcentajes obtenidos durante la investigación, en términos de equidad de género. La cantidad de mujeres vinculadas a la Orquesta anualmente puede variar según el número de estudiantes admitidas y graduadas de la carrera y, del mismo modo, la planta artística puede modificarse varias veces al año.

- Experiencia de mujeres en orquestas:

a. Directoras: La peruana Carmen Moral fue la primera mujer en asumir la dirección titular de una orquesta en Latinoamérica. En el año 1970, siendo la única mujer entre los participantes, obtuvo por concurso público la dirección de la Orquesta Sinfónica del Perú. Sin embargo, esa no fue la primera vez que los peruanos vieron a una mujer en el podio: años atrás, luego de culminar sus estudios en el exterior, la maestra Moral debutó en un concierto de la temporada de verano que hacía el primer elenco nacional en el Campo de Marte.

Imagínate qué repertorio: Leonora No. 3 de Beethoven; un arreglo de Holzmann sobre La Reina de las Hadas de Purcell, La tarde de un fauno, de Debussy; y la segunda parte era la Sinfonía No. 5 de Tchaikovsky. Había gente hasta en los árboles por la curiosidad. (Moral, 2013)

Entre los años 1988 y 1991, asumió el cargo de directora titular de la Orquesta Filarmónica de Bogotá y desde ese entonces ha dirigido más de
450 obras en Europa, Asia y América. Actualmente, es profesora asociada a la cátedra de dirección en Berklee College of Music.

La colombiana Cecilia Espinosa Arango fue nombrada directora titular por primera vez en el año 2000, cuando se creó la Orquesta Sinfónica EAFIT asociada a la misma universidad. Espinosa pertenecía desde años atrás a la planta docente del departamento de música en el énfasis de dirección y había actuado como directora invitada en Cali, Barranquilla, Venezuela y los Estados Unidos de América.

La Sinfónica de EAFIT inició sus labores con un concierto inaugural, que a su vez se convirtió en el primer concierto de Espinosa como directora titular, conformado por obras de Beethoven, Haydn y del compositor Andrés Posada Saldarriaga, actual director del Departamento de Música: "Le comisionamos una obra a Andrés Posada que se denominó Salmo 55 , invitamos a la maestra Blanca Uribe para que tocara un concierto de Beethoven, el No. 3, y cerramos con una Sinfonía de Haydn" (Espinosa, 2013).

Carmen Moral asegura nunca haber experimentado discriminación en el sentido estricto de la palabra, pero hace tres décadas percibía cierta tensión y curiosidad entre el público y los demás miembros de la Orquesta al momento de dirigir: "Al principio era duro. Era una cosa de decir 'oh, una mujer que dirige'. Pero esta especie de novedad fue pasando y ahora encuentras mujeres directoras. ¡Hay muchísimas, más de lo que crees!". Moral afirma que este proceso no es exclusivo del campo musical, se desprende de la evolución que ha tenido el rol femenino en la sociedad, haciéndola cada vez más partícipe de todos los escenarios públicos.

Cecilia Espinosa confiesa haber sentido rechazo por parte de los integrantes de algunas orquestas durante los inicios de su carrera:

Cuando yo fui por primera vez a dirigir la Sinfónica de Colombia, en esa época aún se llamaba así, me enfrenté a un programa que consistía en el Concierto de Copland, con Javier Asdrúbal Vinasco de solista, una obra de Luis Carlos Figueroa y Sinfonía No. 8 de Dvorak. El segundo día 
de trabajo, una de las violistas que tuvo empatía conmigo me dijo que en la orquesta había apuestas, que habían apostado a que ese concierto se me caía y no salía. (Espinosa, 2013)

Las dos directoras coinciden en que este tipo de situaciones segregativas han cesado con el pasar de los años pero que aún se conserva cierta expectativa ante la figura de una mujer en podio. Creen que se espera que sea más masculina en su versión de mando y causa sorpresa su tendencia a ser sutil y maternal en el momento de dar órdenes. "Si una mujer grita, es una histérica, si un hombre grita, ¡qué temperamento!", sostiene Carmen Moral al respecto. Lo que resulta indiscutible para las dos mujeres es que la calidad del trabajo y la excelencia en el momento de dirigir sobrepasan cualquier predisposición de género.

Cecilia Espinosa asegura que aún falta mucho tiempo para que sea posible hablar de equidad de género en la música y que el machismo enraizado en la cultura colombiana desde hace siglos todavía ocasiona secuelas. Uno de los factores determinantes para la superación de este problema, continúa, será la llegada de una mujer a la Presidencia de la República: si la cabeza visible de un Estado es una mujer, la participación femenina sin duda se incrementará. Según Carmen Moral, el proceso de la música en torno a temas de género no ha sido distinto al de ninguna otra disciplina y su constitución, mayoritariamente masculina, responde a temas sociales ajenos a la profesión. Tanto Moral como Espinosa dejan en claro que aún existen desigualdades en el ejercicio de la música profesional, pero que estas han ido disminuyendo con el correr del tiempo.

b. Instrumentistas: Las respuestas de las instrumentistas a las nueve preguntas formuladas en el cuestionario propuesto pueden agruparse en cuatro grupos: discriminación de género, utilización de la imagen femenina en favor del ejercicio musical, desventajas de género en el momento de hacer carrera y el rol femenino en la dirección orquestal. A continuación se revisarán algunas de esas respuestas.
- Discriminación de género: Ninguna de las ocho mujeres encuestadas considera que exista discriminación de género en la música clásica colombiana; sin embargo, cuatro de ellas aseguran que hay una predisposición ante el trabajo femenino en el campo de la interpretación. Dos de ellas afirman que suele preferirse el trabajo masculino porque se desconfía de las capacidades profesionales de las mujeres, mientras que otras dos coinciden en que prevalece en la percepción colectiva el estereotipo que supone un carácter débil en las mujeres y las hace no aptas para ser líderes o solistas, como en el caso de las jefaturas de fila.

- Ninguna de las encuestadas afirma haber sido víctima directa de discriminación de género, pero tres de ellas sostienen haber experimentado rechazo y recelo por parte de sus colegas en más de una ocasión, y haber recibido comentarios que denotan sorpresa al comprobar que sobresalen en sus respectivos instrumentos. Si bien estos comentarios vienen en forma de reconocimientos y felicitaciones, denotan un machismo sutil que permite entrever un aire de sorpresa al obtener tales resultados de una mujer.

- Utilización de la imagen femenina en favor del ejercicio musical: Solamente una de las encuestadas se mostró en desacuerdo con el uso de la imagen femenina en el momento de promocionar su carrera, afirmando que ese tipo de acciones caen en el juego de objetivación de la mujer y repercuten de manera negativa en los avances de género. Las otras siete instrumentistas encuentran perfectamente válido sumar la belleza física, o el hecho de ser mujeres, a atributos como el talento y el profesionalismo para fortalecer un camino laboral.

- Desventajas de género en el momento de hacer carrera: Cuatro encuestadas están en desacuerdo con que es más sencillo hacer 
carrera en la música clásica profesional cuando se es hombre, afirman que si fue así hace algunos años, en este momento el género resulta irrelevante. Las otras cuatro aseguran que por razones históricas en este mundo es más sencillo ser hombre que mujer, pues no se deben enfrentar a obstáculos como la violencia sexual, la mujer como arma de guerra, y la obtención de salarios más bajos; factores que son extensibles al ámbito musical.

- El rol femenino en la dirección orquestal: Ninguna de las mujeres considera a la dirección orquestal como un oficio exclusivo de hombres; sin embargo, todas coinciden en que es un campo mayoritariamente masculino y que esto responde a la cultura machista y patriarcal que ha caracterizado la historia de la sociedad colombiana. La tendencia a asignar virtudes de mando al hombre y de obediencia a la mujer todavía repercute al asumir liderazgos.

\section{Discusión}

\section{Las mujeres y la música sinfónica colombiana}

Indiscutiblemente los resultados arrojados por la investigación aquí presentada demuestran que la participación de mujeres profesionales en la música sinfónica colombiana sigue siendo minoritaria. Para entender esta circunstancia, es necesario remitirse a dos conceptos importantes: la exhibición y el papel del hombre como controlador de la naturaleza según las concepciones patriarcales.

Para Green (2001), aparecer en un espacio compartido por más personas, sentarse en un salón de clase, hacer fila en un supermercado o subirse a un escenario, constituyen actos de exhibición. Ahora bien, dependiendo del contexto en que dicha exhibición tenga lugar podrá catalogarse como institucionalizada, si se realiza frente a un público y representa una separación simbólica con el mismo, o informal, si propone exactamente lo contrario, es decir, la aparición del sujeto en una situación coti- diana. Pronunciar un discurso, actuar en una obra de teatro o ejecutar un instrumento se consideran exhibiciones institucionalizadas, mientras caminar por la calle o asistir al trabajo representan exhibiciones informales.

La historia de la humanidad demuestra que dentro de la exhibición institucionalizada existe una estrecha relación entre el género del sujeto y la connotación que recibe. Según Ramos (2003), la exhibición institucionalizada protagonizada por varones asume un carácter intimidatorio y de poder, como en el caso de las fuerzas militares o los jugadores de rugby, mientras que la exhibición femenina suele relacionarse con ámbitos clandestinos y asociados a la prostitución, como las cantantes de ópera rusa en el siglo XVIII, las bailarinas de Paris durante el siglo XIX y las instrumentistas de jazz en EE. UU. durante el siglo $\mathrm{xx}$.

Por esta razón, las mujeres colombianas estuvieron vedadas ante cualquier tipo de exhibición institucionalizada durante los siglos xix y xx, alejándolas así de los escenarios públicos y convirtiendo sus incursiones musicales en eventos domésticos. Era común que aprendieran instrumentos como parte de su educación básica, sin embargo, su interpretación siempre tenía lugar dentro de la esfera familiar y con repertorios ligeros escritos especialmente para ser interpretados dentro del hogar, como es el caso de los Cuadernos para señoritas compuestos por Roberto Pineda Duque.

En referencia al papel del hombre como controlador de la naturaleza, segundo concepto a tener en cuenta, Lucy Green afirma en su libro:

En el patriarcado, los hombres y las mujeres no sólo desempeñan las funciones prácticas de género, como el tipo de trabajo que realicen, sino que, además, crean y negocian conjuntos de características, marcadas por el género. En su forma de máxima polarización, la masculinidad se define como activa y productiva, comprometida con la búsqueda del saber, y por tanto racional, inventiva, experimental, científica y tecnológica, ligada con la producción del arte y en consecuencia, creativa. En el polo opuesto, la feminidad se define como 
pasiva y reproductora, involucrada en la crianza de otros, y por tanto cuidadora, ocupada en la producción de objetos y servicios útiles, en conclusión, diligente. (Green, 2001, p. 22)

Según esto, el papel del hombre ha sido siempre controlar la naturaleza y el de la mujer ser parte ella. El hombre debe, entonces, manejar la tecnología, elaborarla y dominarla, para así poder intervenir y alterar fenómenos naturales; mientras la mujer, al ser parte de la naturaleza, debe mantenerse alejada de cualquier asomo de tecnología, como, por ejemplo, los instrumentos musicales. En Colombia fueron pocos los instrumentos permitidos a las señoritas de principios del siglo $\mathrm{xx}$, el piano y el arpa fueron considerados aptos, en primer lugar, por su postura sedente, que no atentaba contra la feminidad de sus intérpretes; y, en segundo lugar, por su correspondencia con el ámbito familiar y hogareño, ya que el piano era considerado como un mueble más.

Estas concepciones, desde luego, se han ido aboliendo con el pasar de los años, pero cabe resaltar que Colombia es un país atrasado en este tipo de procesos: mientras la ola mundial de las sufragistas tenía lugar a finales del siglo xIx y comienzos del $\mathrm{xx}$, las mujeres colombianas votaron por primera vez en 1957 y fueron admitidas en la universidad hasta 1935. Así pues, no debe causar asombro que aseveraciones como las de Green aún tengan repercusiones en la mentalidad colombiana.

Cuando una mujer ejecuta un instrumento como parte de una orquesta, está rompiendo con todas las connotaciones asignadas a lo femenino desde conceptos como la exhibición y el aislamiento tecnológico, primero, porque el hecho de aparecer en escena con un instrumento musical desvirtúa su rol de naturaleza controlada y la pone del lado controlador; segundo, porque al hacerlo, su exhibición institucionalizada pierde cualquier tinte sexual y asume un carácter de liderazgo y poder.

En un país como Colombia, donde según las estadísticas presentadas por la Alta Consejería Presidencial para la Equidad de la Mujer (Presidencia de la República s. f.), anualmente ocurren cerca de 1200 homicidios perpetrados contra mujeres, se denuncian cerca de 65000 casos de violencia contra la mujer, y la participación de mujeres en política no supera el $20 \%$; resulta apenas lógico que los procesos de exhibición y control tecnológico de la naturaleza propuestos por Green continúen teniendo alguna vigencia.

Si bien los resultados obtenidos durante la investigación realizada a las cinco orquestas profesionales del país no difieren en mucho de los resultados obtenidos en investigaciones similares realizadas en otros países, la cifra que establece un $30 \%$ de participación femenina en la música sinfónica colombiana y su estatismo durante los últimos diez años demuestra el atraso y la lentitud de los progresos relacionados con asuntos de género en Colombia.

\section{Las mujeres al mando}

La inclusión de las mujeres en la música profesional ha sido un proceso largo y dispendioso, pero lo ha sido aún más, la inclusión de mujeres en espacios de liderazgo y poder. La primera mujer en dirigir una de las llamadas "orquestas de varones" responde a un caso único y aislado en 1930, cuando la holandesa Antonia Bricho debuta con la Filarmónica de Berlín y tras regresar a Estados Unidos, su país de residencia, no vuelve a recibir ningún tipo de propuesta. Cuarenta años más tarde, la periodista estadounidense Judy Collins publica un documental sobre la vida de Bricho que la ubica nuevamente en la escena musical. La pianista británica Ethel Leginska también incursionó como directora durante la primera mitad del siglo $\mathrm{xx}$, sin embargo, las invitaciones no eran muy frecuentes y en repetidas ocasiones tuvo que ofrecerse como solista a cambio de dirigir como invitada (Brydie-Leigh, 2008).

En Colombia, la panista Teresa Tranco compuso una zarzuela de dos actos sobre textos de Carlos Sáenz Echeverría llamada Similia Similibus (Fula, 2012). Fue estrenada en la sala de su casa el 15 de octubre de 1883 y, en los registros existentes, ella aparece como directora de la orquesta que la interpreta. Al poco tiempo, Tranco se casa con Alejandro Herrera, reconocido comerciante de la época, y abandona su actividad musical. El siguiente registro 
de mujeres directoras en la música colombiana data de 1988, cuando Carmen Moral asume la dirección titular de la Orquesta Filarmónica de Bogotá.

La experiencia profesional de las directoras Carmen Moral y Cecilia Espinosa coinciden en que el progreso del país en asuntos de género es evidente, ya no existen políticas sexistas, se ha legislado a favor de la equidad y se condena el abierto rechazo a la mujer en el podio. Sin embargo, siguen presentes una serie de predisposiciones ante el ejercicio de la dirección por mujeres, lo que demuestra que el problema está en la mentalidad social y no en los hechos de facto.

La aparición de una mujer como cabeza visible en una orquesta genera varios choques frente al concepto de exhibición arraigado en las culturas patriarcales. Por un lado, expone la figura femenina sin ningún atenuante, como el uso de instrumentos, por ejemplo; y por el otro, la ubica en una posición de superioridad y liderazgo. De alguna manera, se está atentando contra el principio de alteridad de una cultura como la nuestra, dominada por el machismo durante tantos años.

Una mujer directora enfrenta tres problemas en torno a la forma en que su trabajo es recibido por los demás: el primero de ellos corresponde a su posicionamiento como figura de poder, "aunque en la actualidad se respetan las mujeres al mando, existe una tendencia a esperar menos", afirma Cecilia Espinosa. A menudo se escuchan cumplidos en donde se equipara su trabajo al de los varones, "es tan buena como cualquier hombre" o "no dirige como una mujer", que, si bien nacen a manera de elogio, denotan una discriminación intrínseca. La prensa, por su lado, muchas veces estimula este tipo de sesgos, incluyendo a las directoras en una categoría especial etiquetada como "directoras mujeres", es decir, separando la dirección femenina de la masculina, como si una fuera subcategoría de la otra.

El segundo problema se relaciona estrechamente con el primero, este tipo de expectativas hacen que el nivel de presión de una mujer sea mucho mayor al experimentado por un hombre, pues todo el tiempo se sentirá en la obligación de demostrar que su tra- bajo es igual o superior al de los varones, en especial porque las políticas de participación aprobadas durante los últimos años han alimentado percepciones donde la participación femenina es cuestión de requisito y no de mérito.

El tercer problema se desprende de los dos ya planteados: ¿de qué forma debe enfrentarse el sesgo social de las mujeres en la dirección? Tanto Carmen Moral como Cecilia Espinosa aseguran que lo más indicado es desprenderse de cualquier hegemonía masculina al momento de dirigir, pensar solo en que se es un músico haciendo música y que el género no tiene ningún tipo de repercusión. Esto no quiere decir que se deba dar la espalda a la realidad y desconocer las barreras de género que aún persisten, simplemente, que esta labor debe hacerse por fuera del podio.

\section{Las mujeres y la imagen}

Cualquier discurso musical se compone de dos tipos de significados: intrínsecos, que operan en el marco de las interrelaciones entre materiales musicales, y evocados, que surgen de la relación entre factores contextualizados y simbólicos (Green, 2001). En cuanto a los significados del primer caso, la interpretación es una sola y suele ser la misma para cualquier receptor, mientras que en lo relacionado con los significados del segundo caso, existen múltiples interpretaciones y estas dependen de la experiencia personal que quien escucha. La forma, las notas y el ritmo de una obra son significados intrínsecos, mientras el momento social en que se ejecuta y la apariencia de su intérprete son significados evocados.

Pretender que al hacer música el género es irrelevante resulta bastante ingenuo. La figura femenina, símbolo de sensualidad y belleza desde hace siglos, jamás pasará desapercibida en ningún espacio de exhibición y mucho menos, si esta tiene lugar en un escenario frente a cientos de personas. En definitiva, el género del intérprete, en este caso su feminidad y apariencia física, otorgan una serie de significados evocados a la música que se está ejecutando, sin que este hecho traiga repercusiones negativas o deslegitime procesos de equidad de género necesariamente. 
Una de las primeras mujeres en reconocer y aprovechar la feminidad de manera positiva fue la violonchelista Ofra Harnoy, quien decidió usar su figura femenina como impulsora de la grabación completa que hizo de los conciertos de Vivaldi. En 1989, Harnoy apareció recostada con una mano en la cadera y otra sobre su chelo, en la portada de un disco que grabó con el director Robert Dodson y la Toronto Chamber Orchestra.

Pensamientos como el de Harnoy se han vuelto cada vez más populares. La violinista Vanessa Mae se ha hecho reconocida por hacer uso de su belleza en el momento de publicitar sus discos y varias agrupaciones de cámara se han promocionado como grupos de mujeres. La censura, desde luego, ha sido certera. La pianista Juja Wang, virtuosa y reconocida por la calidad de sus interpretaciones, ha sido fuertemente criticada por usar faldas cortas en el escenario, tanto, que tras un concierto en el Hollywood Bowl de Los Ángeles, durante el 2011, el crítico Mark Swed del LA Times afirmó que, de ser un poco más corta la falda de Wang, habría tenido que prohibirse la entrada a menores de edad. Acusación a la que la pianista respondió con un dejo de humor: "Soy mujer, me gusta la moda” (Morreale, 2014).

Comentarios como los de Swed resultan más frecuentes de lo esperado, incluso existen corrientes de la musicología de género que se muestran en desacuerdo con esta forma de asumir el perfil femenino en la música, las cuales argumentan que es por esta razón que se subestiman las capacidades de la mujer en cualquier oficio, utilizar el cuerpo o la belleza como medios para impulsarse profesionalmente es caer en el juego de la objetivación sexual.

Cabe resaltar, que de las ocho instrumentistas que respondieron al cuestionario aplicado durante esta investigación, siete coincidieron en que la belleza femenina es un valor agregado y que si se explota en conjunto con el talento, la responsabilidad y el profesionalismo, trae muy buenos resultados. Pretender anular los significados evocados de la música y reducirla únicamente a sus relaciones intrínsecas, no es solo una empresa arcaica sino imposible. Cualquier tipo de comunicación está determinada por la imposición que ha tenido la imagen durante los últimos años, negar su relevancia y no usarla a favor de la música y la profesión misma es negarse de alguna manera como intérprete.

\section{Conclusión}

Desde la inclusión de la equidad de género entre los derechos sociales, económicos y culturales de la constitución colombiana en 1991, no es posible hablar de discriminación de género en el sentido estricto de la palabra, sin embargo, la cultura patriarcal que ha regido la historia del mundo desde sus inicios y el machismo cultural que ha tenido cabida durante más de un siglo en nuestro país aún tienen repercusiones en los distintos escenarios de la sociedad.

La música, por supuesto, se encuentra permeada por estas repercusiones. La participación de mujeres en las orquestas profesionales sigue siendo minoritaria, las mujeres en escenarios de poder como la dirección aún se enfrentan a un sinnúmero de prevenciones, la prensa tiende a separar el trabajo masculino del femenino, convirtiendo este último en una subcategoría del primero y las mujeres pertenecientes a este ámbito aseguran haber percibido en más de una ocasión, predisposición, desconfianza y rechazo frente a su trabajo.

El panorama es claro: en la música profesional colombiana no existen políticas sexistas y la discriminación hacia la mujer se condena, pero, indiscutiblemente, se evidencia una marcada tendencia a menospreciar el trabajo femenino y a asumir con prejuicio que su calidad se encuentra por debajo de la de los varones.

La equidad de género aún está lejos, pero de ello no puede culparse enteramente al entorno musical, su desarrollo y evolución en estos temas está subordinado al avance en el resto de ámbitos sociales, es decir, el cambio de mentalidad en la música solo será posible cuando la equidad de género sea premisa inviolable para la sociedad colombiana. 


\section{Referencias}

Brydie-Leigh, B. (2008). You're a woman a our orchestra just won't have you. Hecate: an interdisciplinary journal of women's liberation, 3 (34), 6-23.

Espinosa Arango, C. (6 de Diciembre de 2013). Directoras en la música sinfónica colombiana. (L. M. Galindo Morales, Entrevistador)

Facio, A. (2000). Feminismo, género y patriarcado. Santiago: Lom.

Fula, L. (2012). Repertorio y archivo: El tránsito de las mujeres bogotanas del espacio privado al público a través de la música del siglo xx. En C. Millán de Benavides y A. Quintana (eds.), Mujeres en la música en Colombia. El género de los géneros (pp. 219-242). Bogotá: Editorial Javeriana e Instituto Pensar.

Galeano, M. (2004). Diseño de proyectos en la investigación cualitativa. Medellín, Colombia: Fondo editorial Universidad EAFIT.

Green, L. (2001). Música, Género, Educación. Madrid: Ediciones Morata.

Levine, R. (2009). Age, Gender and Orchestras. Poliphonic.org. Recuperado de http://www.polyphonic.org/article/age-gender-and-orchestras/

Moral, C. (17 de Noviembre de 2013). Directoras en la Música Sinfónica Colombiana. (L. M. Galindo Morales, Entrevistador)
Morreale, M. (2014). Classical music's shocking gender gap. CBCMusic. Recuperado dehttp://music.cbc.ca/\#/ blogs/2014/3/Classical-musics-shocking-gender-gap

Oakley, A. (1972). Sex, gender and society. Londres: Temple Smith.

Padua, J. (1987). Técnicas de investigación aplicadas a las ciencias sociales. México D.F, México: Fondo de Cultura Económica.

Presidencia de la república. (s.f.). Recuperado el 12 de 06 de 2014, de Alta consejería presidencial para la equidad de la mujer: http://www.equidadmujer.gov.co

Programa de las Naciones Unidas para el Desarrollo. (s.f.). Programa de las Naciones Unidas para el Desarrollo en Colombia. Recuperado el 20 de Junio de 2014, de http://www.pnud.org.co/img_upload/196a010e 5069f0db02ea92181c5b8aec/Estrategia\%20de\%20 genero\%20PNUD\%20Colombia.pdf

Ramos, P. (2003). Feminismo y música, introducción crítica. Madrid: Narcea.

Velásquez, J. (2002). El encanto de las damas: las mujeres y la práctica musical a finales de siglo XIX en Medellín, Colombia. En C. Millán de Benavides y A. Quintana (eds.), Mujeres en la música en Colombia. El género de los géneros (pp. 193-218). Bogotá: Editorial Javeriana e Instituto Pensar. 